\title{
Novedades y NueVAS CITAS DE MUSgos (BRyophyta) de la Reserva Natural Punta Lara (Buenos Aires, Argentina)
}

\author{
Novelties AND NeW Moss Records (Bryophyta) FRoM Punta LARA \\ Nature Reserve (Buenos Aires, Argentina)
}

\author{
Fabricio Emanuel Valdés ${ }^{1,2 *} \mathbb{D}$, Denilson Fernandes Peralta ${ }^{3}$, Agustina Celeste \\ Cottet $^{4,5}$ (D) Adolfina Savoretti ${ }^{\text {(DD }}$ y Marta Noemí Cabello ${ }^{1,2}$ (D)
}

1. Instituto de Botánica Spegazzini, Facultad de Ciencias Naturales y Museo, Universidad Nacional de La Plata, calle $53 \mathrm{~N}^{\circ} 477$, La Plata, Buenos Aires, Argentina

2. Comisión de Investigaciones Científicas $(\mathrm{ClC})$ de la Provincia de Buenos Aires, La Plata, Buenos Aires, Argentina

3. Instituto de Botânica, Av. Miguel Estéfano 3687, CEP04301-902, São Paulo, São Paulo, Brasil

4. Instituto de Investigaciones en Biodiversidad y Medioambiente (INIBIOMA); Universidad Nacional del Comahue (UNComahue), Quintral 1250, CP 8400 San Carlos de Bariloche, Río Negro, Argentina 5. Centro Austral de Investigaciones Científicas (CADIC-CONICET), Bernardo Houssay 200, CP9410 Ushuaia, Tierra del Fuego, Argentina

*iam.rondii@gmail.com

\section{Citar este artículo}

VALDÉS, F. E., D. FERNANDES PERALTA, A. C. COTTET, A. SAVORETTI \& M. N. CABELLO. 2021. Novedades y nuevas citas de musgos (Bryophyta) de la Reserva Natural Punta Lara (Buenos Aires, Argentina). Bol. Soc. Argent. Bot. 56: 141-150.

DOI: https://doi. org/10.31055/1851.2372.v56. n2.30132

Recibido: 7 Sep 2020

Aceptado: 12 Abr 2021

Publicado en línea: 3 May 2021

Publicado impreso: 30 Jun 2021

Editor: Juan Larraín (D)

ISSN versión impresa 0373-580X

ISSN versión on-line 1851-2372

\section{SUMMARY}

Background and aims: A list of mosses recorded in Punta Lara Natural Reserve (Buenos Aires, Argentina) is presented, increasing the knowledge of the diversity and distribution of mosses in the country.

M\&M: The specimens were collected and identified according to conventional taxonomic criteria and deposited in the Herbarium of the Spegazzini Institute (LPS). Global Biodiversity Information Facility (GBIF) records were consulted as a source of information about the species distribution.

Results: Fifteen species of mosses distributed in 11 families were identified in Punta Lara Natural Reserve. The genus Thamnomalia Olsson, Enroth \& Quandt, represented by Thamnomalia glabella (Hedw.) Olsson, Enroth \& Quandt and the species Sematophyllum adnatum (Michx.) E. Britton are recorded for the first time for Argentina.

Discussion and Conclusions: Two species are new records for Argentina and 11 species are recorded for the first time for Buenos Aires province. Several species are tropical, extending their distribution towards temperate regions.

\section{KEY WORDS}

Brachytheciaceae, Fissidentaceae, Hookeriaceae, Neckeraceae, Sematophyllaceae, Sematophyllum adnatum, Thamnomalia glabella.

\section{RESUMEN}

Introducción y objetivos: Se presenta una lista de musgos registrados en la Reserva Natural Punta Lara (Buenos Aires, Argentina) con el objetivo de ampliar el conocimiento sobre la distribución de las especies presentes en el país.

M\&M: Los especímenes fueron recolectados e identificados de acuerdo a los criterios taxonómicos convencionales y depositados en el Herbario del Instituto Spegazzini (LPS). Registros del Servicio de Información sobre la Diversidad Biológica Mundial (GBIF) fueron consultados como fuente de información sobre la distribución de las especies.

Resultados: Como resultado de este estudio se identificaron 15 especies de musgos pertenecientes a 11 familias en la Reserva Natural Punta Lara. Se cita por primera vez para Argentina la especie Sematophyllum adnatum (Michx.) E. Britton y el género Thamnomalia Olsson, Enroth \& Quandt, representado por Thamnomalia glabella (Hedw.) Olsson, Enroth \& Quandt.

Discusión y Conclusiones: En este trabajo se registran dos nuevas citas para la Argentina y 11 nuevas menciones para la provincia de Buenos Aires. Varias de las especies recolectadas son tropicales, ampliando su distribución hacia regiones templadas.

\section{Palabras clave}

Brachytheciaceae, Fissidentaceae, Hookeriaceae, Neckeraceae, Sematophyllaceae, Sematophyllum adnatum, Thamnomalia glabella. 


\section{INTRODUCCIÓN}

Las briófitas (Bryophyta sensu lato) son un grupo monofilético (De Sousa et al., 2019) con alrededor de 20.000 especies distribuidas en todos los continentes (Stech \& Quandt, 2014). Dicho grupo está comprendido por antocerotes (Anthocerotophyta), hepáticas (Marchantiophyta) y musgos (Bryophyta sensu stricto), siendo este último el grupo más diversificado (Magill, 2014; Söderström et al., 2016).

Como componentes de la diversidad vegetal las briófitas proveen importantes servicios a los ecosistemas. Son colonizadoras de ambientes disturbados, proporcionan microambientes para el establecimiento y crecimiento de microorganismos, tienen un papel fundamental en el ciclo de nutrientes, la retención de humedad y la disponibilidad de agua, y pueden ser utilizadas como bioindicadores ambientales (Gradstein et al., 2001). Desde el punto de vista de la conservación los datos de su distribución pueden contribuir a entender la historia de la flora briofítica de una región (Delgadillo, 1990).

En Argentina, Matteri (2003) presentó un catálogo con 1500 taxones específicos e infraespecíficos de musgos, entre nombres aceptados y sinónimos. Estudios posteriores han reportado nuevos registros de géneros y especies de musgos para el país (e.g. Suárez et al., 2005; Schiavone \& Suárez, 2007; Fuertes \& Rodríguez, 2008; Schiavone \& Suárez, 2009; Fuertes et al., 2011; Rovere \& Calabrese, 2011; Suárez et al., 2014; Cottet et al., 2016; Colotti et al., 2016; Cano \& Alonso, 2017; Colotti \& Suárez, 2017, 2018, 2019; Colotti et al., 2019; Cottet \& Messuti, 2019; Suárez \& Larraín, 2019; Larraín et al., 2020, Jiménez et al., 2020), los que además se complementan con registros taxonómicos que pueden obtenerse mediante el libre acceso a datos de biodiversidad. Sin embargo, el conocimiento de la diversidad de musgos en algunas áreas, aún es escaso y debe ser profundizado.

La Reserva Natural Punta Lara (RNPL) se encuentra ubicada sobre la costa del Río de La Plata (34 $47^{\prime} \mathrm{S} ; 58^{\circ} 01^{\prime} \mathrm{O}$ ) entre los partidos de Ensenada y Berazategui de la provincia de Buenos Aires, Argentina (Fig. 1). Esta área protegida se encuentra, de acuerdo con Cabrera (1971), dentro la Provincia Pampeana la cual se incluye en el Dominio Chaqueño y la Región Neotropical. Mientras que, según la clasificación de ecorregiones argentinas de Brown \& Pacheco (2005) la RNPL se encuentra en la
"Pampa", originalmente dominada por gramíneas, y en el "Delta e Islas del Paraná", un conjunto de macrosistemas de humedales de origen fluvial. El clima es templado con veranos cálidos, completamente húmedo (Kottek et al., 2006), con una temperatura media anual de $16,6^{\circ} \mathrm{C}$ y precipitaciones medias de $945 \mathrm{~mm}$ (Climate-Data.Org, 2020).

Los estudios florísticos en la RNPL se han centrado principalmente en las plantas vasculares, siendo las briófitas mencionadas sin tratamientos taxonómicos específicos, a excepción del trabajo de Kühnemann (1943) que registra 15 géneros de musgos en la reserva, de los cuales, cuatro fueron identificados a nivel de especie.

Poco se sabe sobre la diversidad taxonómica de las briófitas en la reserva, incluyendo aquellos ambientes por fuera de las selvas marginales (principales ambientes por los cuales se establecieron originalmente políticas de conservación) que han sufrido cambios en el uso del suelo y alteraciones del paisaje mediante la canalización y consecuente formación de albardones artificiales, así como por la introducción de especies exóticas.

En este trabajo se presenta una lista de musgos recolectados en la RNPL, con el objetivo de realizar un estudio sobre la diversidad taxonómica de los mismos, donde se amplía la distribución de especies tradicionalmente conocidas como neotropicales, mencionando novedades para la provincia de Buenos Aires y Argentina.

\section{Materiales y Métodos}

Los especímenes estudiados a través de 20 muestras compuestas (Fig. 1), fueron recolectados entre los meses de marzo-junio de 2018-2019. Los ambientes fueron seleccionados sobre la base de unidades fisonómicas y esquemas vegetacionales establecidos por Herrera \& Torres Robles (2012). El material fue preparado y herborizado de acuerdo a los métodos propuestos por Frahm (2003). La identificación de los especímenes se realizó siguiendo los criterios taxonómicos de Demaret \& Empain (1985), Ireland (1992), Churchill \& Linares (1995), He (1997), Hedenäs (2003), Pursell (2007) y Fuertes \& Marchessi (2013). La clasificación a nivel de familia se estableció según Goffinet \& Buck (2004). El material examinado se encuentra depositado en el Herbario del Instituto Spegazzini (LPS). Los Registros del Servicio de 


\section{F. E. Valdés et al. - Musgos de la Reserva Natural Punta Lara}

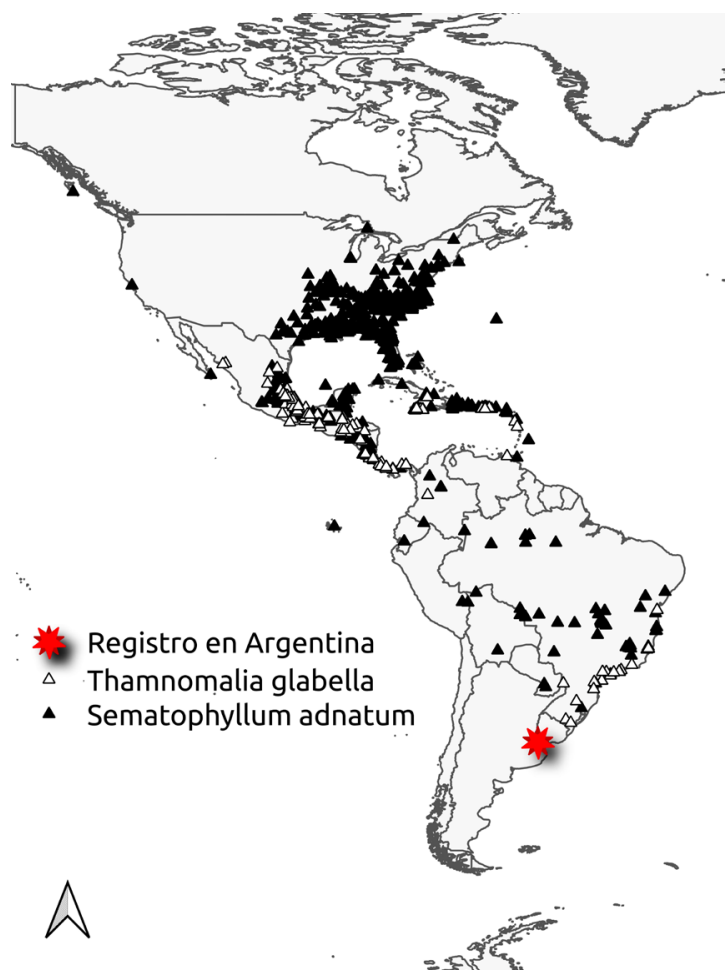

Fig. 1. Ubicación de la Reserva Natural Punta Lara. Se indican los sitios de recolección de muestras (estrella roja) y las distribuciones mundiales de los nuevos registros para la Argentina aquí reportados. (SRC: WGS 84).

Información Sobre la Diversidad Biológica Mundial (GBIF) se utilizaron como fuente de información sobre la distribución de especies, utilizando sólo aquellos registros en los cuales los especímenes hayan sido revisados por especialistas.

\section{Resultados}

A partir de las muestras recolectadas se determinaron 15 especies de musgos pertenecientes a 11 familias. Dos especies son nuevas citas para la Argentina y 11 corresponden a nuevas menciones para la provincia de Buenos Aires. A continuación, se presentan las especies en orden alfabético y la familia a la que pertenece entre corchetes. Las especies que se presentan como nuevas citas para Argentina están precedidas por un asterisco $\left(^{*}\right)$ acompañadas por descripciones e ilustraciones.
Brachythecium austroglareosum (Müll. Hal.) Kindb. [Brachytheciaceae]

Distribución. Esta especie se encuentra a lo largo de la zona andina de América del Sur, alcanzando su distribución más austral en Tierra del Fuego, Antártida e Islas del Atlántico Sur, encontrándose además en Nueva Zelanda (McFarland, 1989). En Argentina ha sido registrada en las provincias de Chubut, Santa Cruz y Tierra del Fuego, Antártida e Islas del Atlántico Sur (Matteri, 2003). Se cita por primera vez para la provincia de Buenos Aires.

Comentarios. Los miembros de la familia Brachytechiaceae presentan una gran plasticidad fenotípica la cual es afectada por las condiciones del ambiente y se manifiesta en notables variaciones morfológicas (Draper \& Hedenäs, 2009).

Material estudiado. ARGENTINA. Prov. Buenos Aires: Berazategui. Reserva Natural Punta Lara, suelo de vegetación exótica, 2018, Valdés 6 (LPS 49129); sobre albardón de vegetación exótica, 2019, Valdés 20 (LPS 49143).

Campylopus flexuosus (Hedw.) Brid. [Leucobryaceae]

Distribución. Esta especie se ha mencionado como un endemismo Gondwánico, restringido a la Región Neo- y Paleotropical (Stech \& Wagner, 2005). En Argentina ha sido registrada en la provincia de Tierra del Fuego, Antártida e Islas del Atlántico Sur (Matteri, 2003). Se cita por primera vez para la provincia de Buenos Aires.

Material estudiado. ARGENTINA. Prov. Buenos Aires: Berazategui. Reserva Natural Punta Lara, suelo de vegetación exótica, 2018, Valdés 6 (LPS 49129); sobre corteza de vegetación exótica, 2019, Valdés 11 (LPS 49134).

\section{Cyclodictyon albicans (Hedw.) Kuntze} [Pilotrichaceae]

Distribución. Esta especie está ampliamente distribuida en la Región Neotropical (Churchill \& Linares, 1995). En Argentina ha sido registrada en 
la provincia de Misiones (Matteri, 2003). Se cita por primera vez para la provincia de Buenos Aires.

Comentarios. Kühnemann (1943) cita e ilustra un espécimen de Punta Lara como Cyclodictyon sp., al cual compara con Hookeria uliginosa Müll. Hal.

Material estudiado. ARGENTINA. Prov. Buenos Aires: Ensenada. Reserva Natural Punta Lara, selva marginal, 2019, Valdés 13 (LPS 49136).

Distichophyllum rotundifolium (Hook.f. \& Wilson) Müll.Hal. \& Broth. [Daltoniaceae]

Distribución. Esta especie se encuentra en Argentina, Chile, Sudeste de Australia y Nueva Zelanda (Müller, 2019). En Argentina ha sido registrada en la provincia de Río Negro (Matteri, 2003). Se cita por primera vez para la provincia de Buenos Aires.

Material estudiado. ARGENTINA. Prov. Buenos Aires: Ensenada. Reserva Natural Punta Lara, suelo de vegetación de especies exóticas, 2019, Valdés 12 (LPS 49135).

\section{Fissidens elegans Brid. [Fissidentaceae]}

Distribución. Esta especie se encuentra ampliamente distribuida en la Región Neotropical (Pursell, 2007). En Argentina ha sido registrada en las provincias de Corrientes, Misiones (Matteri, 2003), Jujuy y Salta (Pursell, 2007). Se cita por primera vez para la provincia de Buenos Aires.

Material estudiado. ARGENTINA. Prov. Buenos Aires: Ensenada. Reserva Natural Punta Lara, suelo de vegetación de especies exóticas, 2019, Valdés 12 (LPS 49135).

\section{Fissidens zollingeri Mont. [Fissidentaceae]}

Distribución. Esta especie se encuentra ampliamente distribuida en la Región Neotropical, sur de los Estados Unidos, Asia, Australia, Malasia, Oceanía y África tropical (Pursell, 2007). En Argentina ha sido registrada en las provincias de Corrientes, Jujuy,
Misiones, Tucumán (Pursell, 2007) y Salta (Magill et al., 2020). Se cita por primera vez para la provincia de Buenos Aires.

Material estudiado. ARGENTINA. Prov. Buenos Aires: Ensenada. Reserva Natural Punta Lara, sobre albardón de vegetación nativa, 2018, Valdés 3 (LPS 49126); Valdés 4 (LPS 49127).

Helicodontium capillare (Hedw.) A.Jaeger [Brachytheciaceae]

Distribución. Esta especie se encuentra ampliamente distribuida en la Región Neotropical a bajas altitudes (Churchill \& Linares, 1995). En Argentina ha sido registrada en las provincias de Chaco, Corrientes, Entre Ríos, Formosa y Misiones (Matteri, 2003). Se cita por primera vez para la provincia de Buenos Aires.

Material estudiado. ARGENTINA. Prov. Buenos Aires: Ensenada. Reserva Natural Punta Lara, selva marginal, 2018, Valdés 7 (LPS 49130); Valdés 8 (LPS 49131); bosque de especies exóticas, 2018, Valdés 9 (LPS 49132); suelo de vegetación de especies exóticas, 2019, Valdés 12 (LPS 49135). Berazategui. Reserva Natural Punta Lara, bosque ribereño, 2019, Valdés 18 (LPS 49141).

Hygroamblystegium varium (Hedw.) Mönk. [Amblystegiaceae]

Distribución. Esta especie se encuentra ampliamente distribuida en América, zonas templadas de Asia y Europa, llegando a regiones subtropicales y en el norte de África y Australia (Hedenäs, 2003). En Argentina ha sido registrada en las provincias de Buenos Aires, Neuquén, Tierra del Fuego, Antártida e Islas Australes del Atlántico Sur (Matteri, 2003) y Jujuy (Hedenäs, 2003).

Comentarios. Para esta especie se utilizó el nombre de Hygroamblystegium varium, que hace referencia a Amblystegium de zonas inundables e incluiría la unificación de muchas especies de Amblystegium (Vanderpoorten \& Hedenäs, 2009). Sin embargo, para la determinación de este espécimen se utilizó la taxonomía propuesta por Hedenäs (2003), que la establece como un sinónimo de Amblystegium varium. 


\section{F. E. Valdés et al. - Musgos de la Reserva Natural Punta Lara}

Material estudiado. ARGENTINA. Prov. Buenos Aires: Ensenada. Reserva Natural Punta Lara, sobre albardón de vegetación exótica, 2019, Valdés 13 (LPS 49136). Berazategui. Reserva Natural Punta Lara, bosque ribereño, 2019, Valdés 19 (LPS 49142).

\section{Isopterygium tenerum (Sw.) Mitt. [Pylaisiadelphaceae]}

Distribución. Esta especie se encuentra ampliamente distribuida en la Región Neotropical, siendo la más común de las ocho especies reportadas para el género (Ireland, 1992). Las especies sudafricanas Plagiothecium rhynchostegioides Müll. Hal., P. sphagnadelphus Müll. Hal. e Isopterygium brachycarpum Dixon, se han reportado como sinonimias de I. tenerum (Ochyra \& Ireland, 2004), ampliando la distribución de esta especie en la Región Paleotropical. En Argentina ha sido registrada en las provincias de Corrientes, Entre Ríos, Misiones, Tierra del Fuego, Antártida e Islas del Atlántico Sur y Tucumán (Matteri, 2003). Se cita por primera vez para la provincia de Buenos Aires.

Material estudiado. ARGENTINA. Prov. Buenos Aires: Ensenada. Reserva Natural Punta Lara, Suelo de vegetación de especies exóticas, 2019, Valdés 12 (LPS 49135).

\section{Ptychostomum pseudotriquetrum (Hedw.) Spence \& H.P.Ramsay [Bryaceae]}

Distribución. Esta especie presenta una amplia distribución en climas templados y fríos del hemisferio norte (Holyoak \& Hedenäs, 2006), Antártida (Okitsu et al., 2003) y tropicales de zonas montañosas de América del Sur (Churchill \& Linares, 1995). En Argentina ha sido registrada en las provincias de Buenos Aires, Chubut, Córdoba, Jujuy, Neuquén, Río Negro, Santa Cruz y Tierra del Fuego, Antártida e Islas Australes del Atlántico Sur (Matteri, 2003).

Material estudiado. ARGENTINA. Prov. Buenos Aires: Ensenada. Reserva Natural Punta Lara, suelo inundado, 2018, Valdés 1 (LPS 49124); suelo sobre albardón de vegetación nativa, 2018, (LPS 49125).
Rhynchostegium serrulatum (Hedw.) A.Jaeger [Brachytheciaceae]

Distribución. Esta especie presenta una amplia distribución en climas templados y tropicales de América (Churchill \& Linares, 1995). En América del Sur se distribuye en los Andes, el sudeste de Brasil, Uruguay y Argentina (McFarland, 1994; Buck, 1998; Fuertes \& Marchessi, 2013; Cottet \& Messuti, 2019). En Argentina ha sido registrada en la provincia de Chubut (Cottet \& Messuti, 2019). Se cita por primera vez para la provincia de Buenos Aires.

Material estudiado. ARGENTINA. Prov. Buenos Aires: Ensenada. Reserva Natural Punta Lara, sobre corteza de vegetación exótica, 2019, Valdés 14 (LPS 49137); Valdés 15 (LPS 49138).

\section{(*) Sematophyllum adnatum (Michx.) E.Britton} [Sematophyllaceae] (Fig.2)

Gametofitos color verde brillante cubriendo la superficie de corteza como un entrelazado laxo. Tallos patentes, con ramificaciones irregulares de terminaciones ascendentes recurvados de 1-2 $\mathrm{cm}$. Filidios lanceolados de hasta de 1,2 $\mathrm{mm}$ de longitud. Ápices de los filidios acuminados. Filidios de márgenes entero-recurvados, ecostados. Células de la lámina linear-fusiformes, lisas y de paredes delgadas. Células alares en tres filas hialinas a pardo-amarillentas. Esporofito con setas rojo-amarillentas de 4-5 mm de longitud. Cápsulas cilíndricas a ovoides. Opérculos largamente cónicos. Caliptras verdes, cuculadas, lisas. Peristoma doble, endostoma hialino, exostoma amarronado, con 16 dientes estriados con línea media en zig-zag.

Distribución. Esta especie se distribuye en América del Norte, Sur y en África tropical (Britton, 1902). Si bien los registros en la Región Neotropical son frecuentes, éstos llegan por el sur hasta el sudeste de Brasil. Se cita por primera vez para Argentina.

Material estudiado. ARGENTINA. Prov. Buenos Aires: Ensenada. Reserva Natural Punta Lara, bosque de especies exóticas, 2018, Valdés 9 (LPS 49132). 


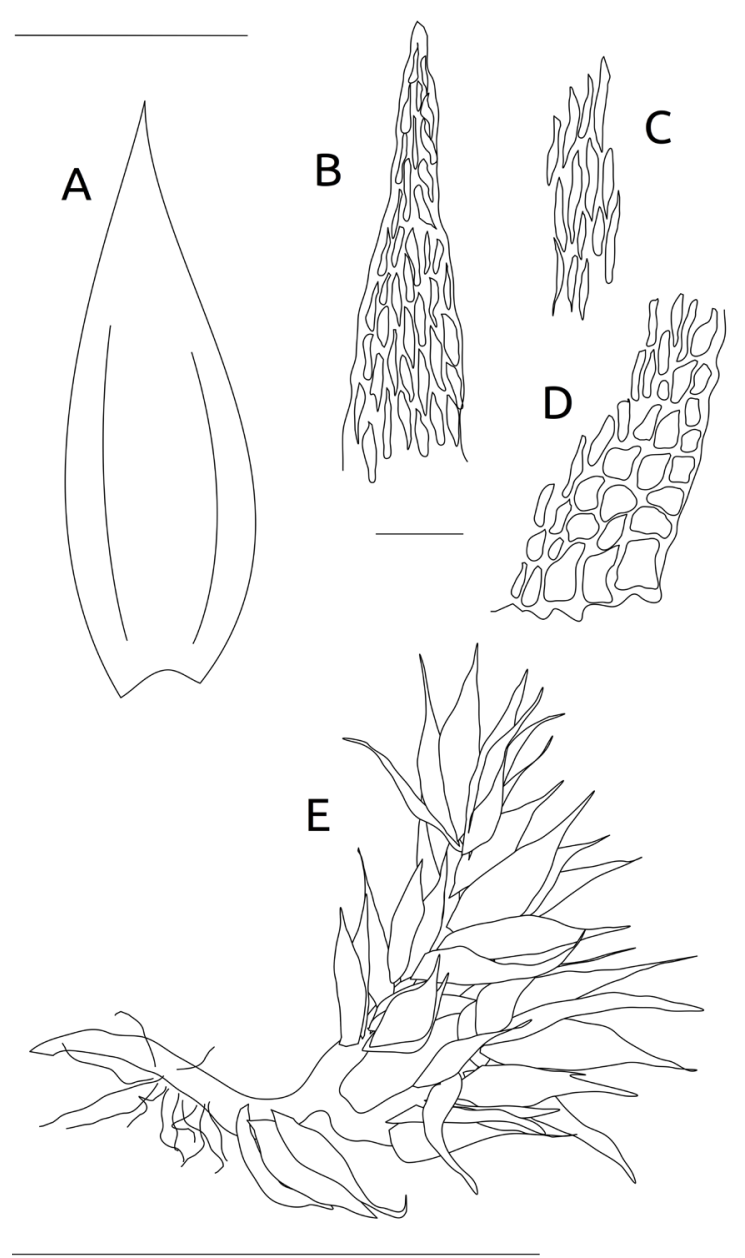

Fig. 2. Sematophyllum adnatum (Michx.) E. Britton. A: Filidio. B: Ápice del filidio. C: Células de la lámina D: Células del ala. E: Gametofito con ramificaciones de terminaciones ascendentes. Escalas $=A: 0,5$ mm; B-D: $250 \mu \mathrm{m}$; E: $250 \mathrm{~mm}$.

\section{Sematophyllum subpinnatum (Brid.) E.Britton [Sematophyllaceae]}

Distribución. Esta especie se encuentra ampliamente distribuida en la región neotropical (Churchill \& Linares, 1995). En Argentina se ha registrado en las provincias de Córdoba, Corrientes y Misiones (Matteri, 2003). Se cita por primera vez para la provincia de Buenos Aires.

Material estudiado. ARGENTINA. Prov.
Buenos Aires: Ensenada. Reserva Natural Punta Lara, bosque de especies exóticas, 2018, Valdés 9 (LPS 49139).

(*) Thamnomalia glabella (Hedw.) S.Olsson, Enroth \& D.Quandt [Neckeraceae] (Fig.3)

Gametofitos de color verde nacarado. Tallo primario rastrero. Tallos secundarios postrados irregularmente ramificados, con ramificaciones postradas a suberectas. Pseudoparafilios presentes, filamentosos hialinos de 100-120 $\mu \mathrm{m}$, con 1 o 2 células basales. Rizoides lisos de color anaranjado. Filidios del tallo primario, oblongo-ovados de $0,75 \times 0,275 \mathrm{~mm}$; filidios del tallo secundario y ramificaciones $1,12 \times 0,5$ $\mathrm{mm}$, ampliamente extendidos, oblongos a oblongoespatulados, asimétricos, algo estrechos en la base y de ápice mucronado, claramente aserrado. Costa corta, bifurcada, una de las bifurcaciones poco visible alcanzando $1 / 10$ y la otra hasta $1 / 3$ de la longitud de la lámina. Células alares oblongo-rectangulares de 18,5 $\times 5 \mu \mathrm{m}$ de paredes moderadamente gruesas. Células basales largo-rectangulares de $26 \times 6 \mu \mathrm{m}$, a linearfusiformes o romboidales alargadas hacia la mitad de la lámina de $25 \times 4,5 \mu \mathrm{m}$. Células del ápice de 12,5× $7,5 \mu \mathrm{m}$, de paredes gruesas. Esporofito no observado.

Distribución. Esta especie se distribuye exclusivamente en regiones tropicales y subtropicales de América Central, sureste de Brasil y Paraguay (Olsson et al., 2011; Cañiza et al., 2016). Se cita por primera vez para Argentina.

Comentarios. El espécimen se encontró fragmentado $(3-4 \mathrm{~cm})$ y escasamente entremezclado con Helicodontium capillare, el cual es dominante sobre la muestra compuesta. Por tal razón, no fue posible determinar el tamaño real de los individuos. La descripción del espécimen estudiado concuerda con las presentadas por He (1997), aunque no se encontró fértil. Esta especie es similar a la ilustración de Neckera villae-ricae Besch. (como Neckeropsis villae-ricae) reportada por Kühnemann (1943) en Punta Lara. Sin embargo, las diferencias se encuentran en el ápice aserrado de los filidios y la costa bifurcada.

Material estudiado. ARGENTINA. Prov. Buenos Aires: Ensenada. Reserva Natural Punta Lara, selva marginal, 2018, Valdés 8 (LPS 49131). 


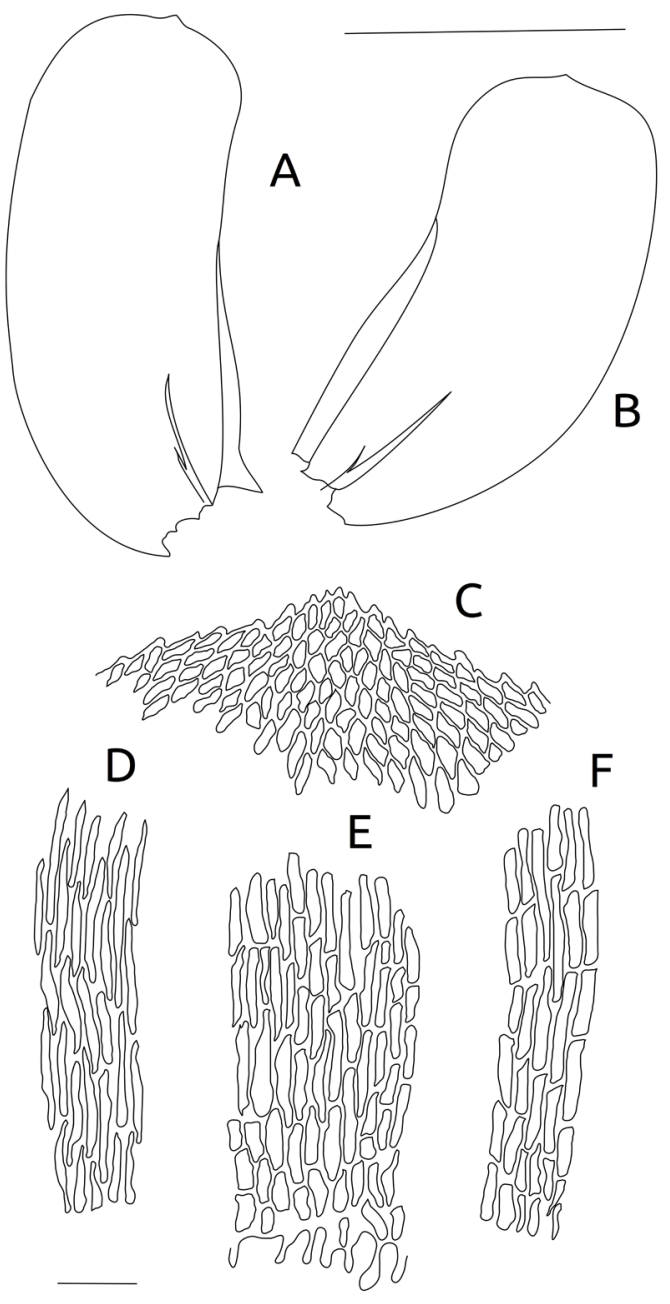

Fig. 3. Thamnomalia glabella (Hedw.) Olsson, Enroth \& Quandt. A-B: Filidios de las ramificaciones secundarias. C: Ápice. D: Células de la mitad de la lámina. E: Células basales cerca de la costa. F: Células del ala. Escalas= A-B: 0,5 mm; C-F: $25 \mu \mathrm{m}$.

Trichostomum brachydontium Bruch. [Pottiaceae]

Distribución. Esta especie presenta una distribución cosmopolita. En Argentina ha sido registrada en la Provincia de Córdoba (Magill et al., 2020) y Salta (Matteri, 2003). Se cita por primera vez para la provincia de Buenos Aires.

Material estudiado. ARGENTINA. Prov. Buenos Aires: Berazategui. Reserva Natural Punta
Lara, sobre albardón de vegetación nativa, 2019, Valdés 5 (LPS 49128).

\section{Discusión y Conclusiones}

En este trabajo se amplía el conocimiento sobre la distribución de musgos neotropicales en Argentina, contribuyendo al estudio de su diversidad. Como resultado fueron identificadas 15 especies de las cuales 2 son nuevas citas para la Argentina y 11 representan nuevos registros para la provincia de Buenos Aires. Las especies Hygroamblystegium varium y Ptychostomum pseudotriquetrum habían sido registradas previamente en la provincia de Buenos Aires, bajo los nombres Orthotheciella varia (Hedw.) Ochyra y Bryum pseudotriquetrum (Hedw.) Gaertn., Mey. \& Scherb. respectivamente. Kühnemann (1943) registró varios especímenes de géneros Bryum, Cyclodictyon, Helicodontium y Rhynchostegium en la provincia, algunos recolectados en Punta Lara. Los mismos se registraron en nuestro trabajo y determinaron a nivel de especie. Debido a esto, varias de las especies resultan nuevas para la provincia, aunque no pudimos corroborar que sean las mismas especies que Kühnemann (1943). Sematophyllum adnatum y T. glabella, son novedades para el territorio argentino. Ambas con una distribución neotropical con registros que llegan hasta el sudeste de Brasil y Paraguay, aunque no han sido registradas en las provincias del litoral argentino.

Dado que Argentina cuenta con un extenso territorio, que además incluye territorios fitogeográficos muy diversos, es necesario explorar áreas intermedias para así incrementar el conocimiento de la flora de briófitas. Nuestros resultados muestran la importancia de realizar estudios florísticos que tienen como objetivo conocer la diversidad de musgos de ambientes aún escasamente explorados. Algunas menciones que parecen importantes remarcar aquí son: (1) que muchas de las especies registradas aquí podrían ser dispersadas por diásporas provenientes de zonas tropicales del sudeste de Brasil y litoral argentino, a través del sistema fluvial del Plata, el cual actúa como una ruta de transporte de plantas trepadoras y epífitas (Guerrero et al., 2018); (2) aquellas especies endémicas $\mathrm{y} / \mathrm{o}$ de provincias alejadas (Región Andino-patagónica) podrían 
ser dispersadas como consecuencia de la acción antrópica, pero la falta de colecciones, acceso y registros de muchas de las provincias argentinas no permiten corroborarlo y; (3) es relevante destacar y profundizar el conocimiento sobre la distribución de aquellas especies que podrían ser utilizadas como indicadoras del estado de conservación de un ambiente.

\section{Agradecimientos}

Al equipo de Guardaparques de la Reserva Natural Punta Lara, por la logística de la campaña realizada para la recolección de las muestras, y por otorgar los recursos y equipamientos necesarios para la movilización. Este estudio fue parcialmente financiado por la Agencia de Promoción Científica y Tecnológica (PICT 0677-Préstamo BID), CICPBA y la Universidad Nacional de La Plata (11 / N 903).

\section{Bibliografía}

BRITTON, E. G. 1902. The genus Sematophyllum. Bryologist 5: 64-66. https://doi.org/10.1639/00072745(1902)5[64:TGS]2.0.CO;2

BROWN, A. D. \& S. PACHECO. 2005. Propuesta de actualización del mapa ecorregional de la Argentina. En: BROWN, A. D., U. MARTÍNEZ ORTIZ, M. ACERBI \& J. CORCUERA (eds.), La situación ambiental argentina 2005, pp. 28-31. Fundación Vida Silvestre, Buenos Aires.

BUCK, W. R. 1998. Pleurocarpous mosses of the West Indies. Mem. N. Y. Bot. Gard. 82: 1-400.

CABRERA, A. L. 1971. Fitogeografía de la República Argentina. Bol. Soc. Argent. Bot. 14: 1-42.

CANO, M. J. \& M. ALONSO. 2017. Three new mosses records from the Andes of northwestern Argentina. Bol. Soc. Argent. Bot. 52: 261-263. https://doi.org/10.31055/1851.2372.v52.n2.17440

CAÑIZA, B. D., D. F. PERALTA, \& G. M. SUÁREZ. 2016. New records and range extension of bryophytes for Paraguay. Cryptogam. Bryol. 38: 393-410. https://doi.org/10.7872/cryb/v38.iss4.2017.393

CHURCHILL, S. P. \& E. L. LINARES C. 1995. Prodromus bryologiae novo-granatensis: Introducción a la flora de musgos de Colombia. Santa Fe de Bogotá, Colombia.
CLIMATE-DATA.ORG2020. Clima: Punta Lara [online] Disponible en: http://es.climate-data.org/ location/764839/ [Acceso: 20 Junio 2020].

COLOTTI, M. T., \& G. M. SUAREZ. 2017. Novelties about the Ditrichaceae in the upper floors of subtropical Yungas from Argentina. Bol. Soc. Argent. Bot. 52: 277-289. https://doi.org/10.31055/1851.2372.v52.n2.17443

COLOTTI, M. T. \& G. M. SUÁREZ. 2018. Sobre la presencia de Mittenothamnium reduncum (Hypnaceae) en la Argentina. Bol. Soc. Argent. Bot. 53: $17-20$. https://doi.org/10.31055/1851.2372.v53.n1.19859

COLOTTI, M. T. \& G. M. SUÁREZ. 2019. Primer registro de Catagonium brevicaudatum (Catagoniaceae) para la Argentina. Bol. Soc. Argent. Bot. 54: 13-16. https://doi.org/10.31055/1851.2372.v54.n1.23575

COLOTTI, M. T., G. M. SUÁREZ \& D. F. PERALTA. 2019. Pleuridium tucumanensis sp. nov. (sección Pleuridium: Ditrichaceae) de Argentina. Revista Mex. Biodivers. 90: 1-5. https://doi.org/10.22201/ib.20078706e.2019.90.2773

COLOTTI, M. T., G. M. SUÁREZ \& M. M. SCHIAVONE. 2016. Novedades sobre la familia Pilotrichaceae para las Yungas de la Argentina. Darwiniana 4: 252-262. https://doi.org/10.14522/darwiniana.2016.42.720

COTTET, A. C., G. M. SUAREZ, M. M. SCHIAVONE \& M. I. MESSUTI. 2016. Cryphaea consimilis (Cryphaeaceae, Bryophyta), nueva cita para la Argentina. Bol. Soc. Argent. Bot. 51: 219-222. https://doi.org/10.31055/1851.2372.v51.n2.14834

COTTET, A. C. \& M. I. MESSUTI. 2019. Dos nuevos registros de musgos (Bryophyta) para la Argentina. Bol. Soc. Argent. Bot. 54: 487-491. https://doi.org/10.31055/1851.2372.v54.n4.24114

DELGADILlO M., C. 1990. Manual de briofitas. Cuadernos del Instituto de Biología 8, UNAM. México.

DEMARET, F. \& A. EMPAIN. 1985. Bryum pseudotriquetrum (Hedw.) Gaert., Meyer \& Scherb. et sa variété bimum (Schreb.) Lilj. Bull. Jard. Bot. Natl. Belg. 55: 275-290. https://doi.org/10.2307/3668019

DRAPER, I. \& L. HEDENÄS. 2009. Circumscription of European taxa within the Sciuro-hypnum reflexum complex (Brachytheciaceae, Bryophyta), based on molecular and morphological data. Taxon 58: 572584. https://doi.org/10.1002/tax.582021

FRAHM, J. P. 2003. Manual of tropical bryology. Trop. Bryol. 23: 1-195. https://doi.org/10.11646/bde.23.1.1 


\section{F. E. Valdés et al. - Musgos de la Reserva Natural Punta Lara}

FUERTES, E. \& M. RODRÍGUEZ. 2008. Aportaciones a la flora briológica argentina. Notula I. Bot. Complut. 32: 75-83.

FUERTES, E., C. SAN MARTÍN \& L. ESCOBAR. 2011. Zelometeorium y Meteoridium (Bryophyta, Brachytheciaceae) en Argentina. Bot. Complut. 35: 27-37. https://doi.org/10.5209/rev_BOCM.2011.v35.3

FUERTES, E. \& J. E. MARCHESSI. 2013. Notula taxonómica, nomenclatural y corológica de Steerecleus (Bryophyta: Brachyteciaceae) en Uruguay. Bot. Complut. 37: 35-39.

http://dx.doi.org/10.5209/rev_BOCM.2013.v37.42266

GOFFINET, B. \& W. R. BUCK. 2004. Systematics of the Bryophyta (mosses): from molecules to a revised classification. Mol. System. Bryophyt. Monogr. System. Bot. 98: 205-239.

GRADSTEIN, S. R., S. P. CHURCHILL \& N. SALAZAR-ALLEN. 2001. Guide to the bryophytes of tropical america. Mem. N. Y. Bot. Gard. 86: $1-577$.

GUERRERO, E. L., M. J. APODACA, F. D. DOSILHIRIART \& P. A. CABANILLAS. 2018. Análisis biogeográfico de los humedales del sistema fluvial del Río de la Plata basado en plantas trepadoras y epífitas. Revista Mex. Biodivers. 89: 1190-1200. https://doi.org/10.22201/ib.20078706e.2018.4.2519

HE, S. 1997. A revision of Homalia (Musci: Neckeraceae). J. Hattori Bot. Lab. 81: 1-52.

HEDENÄS, L. 2003. Amblystegiaceae (Musci). Fl. Neotrop., Monogr. 89: 1-107.

HERRERA, R. \& S. TORRES ROBLES. 2012. Comunidades vegetales de la Reserva Natural Punta Lara. En: ROESLER, I. \& M. G. AGOSTINI (eds.), Inventario de los Vertebrados de la Reserva Natural Punta Lara, provincia de Buenos Aires, Argentina. Temas de Naturaleza y Conservación. Monografía de Aves Argentinas 8: 35-42.

HOLYOAK, D. T. \& L. HEDENÄS. 2006. Morphological, ecological and molecular studies of the intergrading taxa Bryum neodamense and B. pseudotriquetrum (Bryopsida: Bryaceae). J. Bryol. 28: 299-311. https://doi.org/10.1179/174328206X136304

IRELAND, R. R. 1992. The moss genus Isopterygium (Hypnaceae) in Latin America. Trop. Bryol. 6: 11132. https://doi.org/10.11646/bde.6.1.13

JIMÉNEZ, S., G. M. SUÁREZ, CABRAL R. A. 2020. Nuevos registros de musgos del Bosque Chaqueño Seco en Santiago del Estero, Argentina. Bol. Soc. Argent. Bot. 55: 547-555.

https://doi.org/10.31055/1851.2372.v55.n4.30127
KOTTEK, M., J. GRIESER, C. BECK, B. RUDOLF \& F. RUBEL. 2006. World map of the Köppen-Geiger climate classification updated. Meteorol. Zeitsch. 15: 259-263.

https://doi.org/10.1127/0941-2948/2006/0130

KÜHNEMANN, O. 1943. Estudio morfológico y sistemático de los géneros de briofitas de los alrededores de Buenos Aires. Tesis Doctoral, Facultad de Ciencias Exactas y Naturales. Universidad de Buenos Aires, Argentina.

LARRAÍN, J., G. M. SUÁREZ, J. C. BRINDA, R. TANGNEY, M. VON KONRAT \& D. CRABTREE. 2020. Notocynodontium parvulum, a new genus and species of the Rhabdoweisiaceae (Bryophyta: Dicranidae) from southern South America. Nova Hedwigia 150: 97-108.

https://doi.org/10.1127/nova-suppl/2020/097

MAGILL, R. E. 2014. Moss diversity: new look at old numbers. Phytotaxa 9: 167-174.

https://doi.org/10.11646/phytotaxa.9.1.9

MAGILL, B., J. SOLOMON \& H. STIMMEL. 2020. Tropicos Specimen Data. Missouri Botanical Garden. Occurrence dataset [online]. Disponible en: https:// doi.org/10.15468/hja69f [Acceso: Junio 2020].

MATTERI, C. M. 2003. Los musgos (Bryophyta) de Argentina. Bryophyt. Biodivers. Evol. 24: 33-100. https://doi.org/10.11646/bde.24.1.8

MCFARLAND, K. D. 1989. Revision of Brachythecium (Musci) for Mexico, Central America, South America, Antarctica, and circum- Subantarctic islands. Facsimile of the dissertation. Tesis doctoral. University of Tennessee. Estados Unidos.

MCFARLAND, K. D. 1994. Rhynchostegium. In: SHARP, A. J., H. CRUM \& P. M. ECKEL (eds.), The Moss Flora of Mexico, pp. 932-941. The New York Botanical Garden, New York.

MÜLLER, C. 2019. Distichophyllum rotundifolium. GBIF Backbone Taxonomy. Checklist dataset. Disponible en: https://www.gbif.org/species/5280076 [acceso: 20 junio 2020].

OCHYRA, R. \& R. R. IRELAND. 2004. Isopterygium tenerum, newly recognized for Africa. Bryologist 107: 363-367. https://doi.org/10.1639/00072745(2004)107[0363:ITNRFA]2.0.CO;2

OLSSON, S., J. ENROTH, V. BUCHBENDER, L. HEDENÄS, S. HUTTUNEN \& D. QUANDT. 2011. Neckera and Thamnobryum (Neckeraceae, Bryopsida): Paraphyletic assemblages. Taxon 60: 36-50. https://doi.org/10.1002/tax.601005 
OKITSU, S., S. IMURA, \& E. AYUKAWA. 2003. Structure and dynamics of the Ceratodon purpureusBryum pseudotriquetrum community in the Yukidori Valley, Langhovde, continental Antarctica. Polar Biosci. 16: 49-60.

PURSELL, R. A. 2007. Fissidentaceae. Fl. Neotrop., Monogr. 101: 1-279.

ROVERE, A. E. \& G. M. CALABRESE. 2011. Diversidad de musgos en ambientes degradados sujetos a restauración en el Parque Nacional Lago Puelo (Chubut, Argentina). Revista Chilena Hist. Nat. 84: 571-580. https://doi.org/10.4067/S0716-078X2011000400009

SCHIAVONE, M. M. \& G. M. SUÁREZ. 2007. Las Thuidiaceae en el noroeste de Argentina. Bol. Soc. Argent. Bot. 42: 211-230.

SCHIAVONE, M. M. \& G. M. SUÁREZ. 2009. Globulinella halloyi (Pottiaceae), a new species from Argentina. Bryologist 112: 584-588. https://doi.org/10.1639/0007-2745-112.3.584

DE SOUSA, F., P. G. FOSTER, P. C. DONOGHUE, H. SCHNEIDER \& C. J. COX. 2019. Nuclear protein phylogenies support the monophyly of the three bryophyte groups (Bryophyta Schimp.). New Phytol. 222: 565-575. https://doi.org/10.1111/nph.15587

SÖDERSTRÖM, L., A. HAGBORG, A. VON KONRAT et al. 2016. World checklist of hornworts and liverworts. PhytoKeys 59: 1-828. https://doi.org/10.3897/phytokeys.59.6261
STECH, M. \& D. WAGNER. 2005. Molecular relationships, biogeography, and evolution of Gondwanan Campylopus species (Dicranaceae, Bryopsida). Taxon 54: 377-382. https://doi.org/10.2307/25065366

STECH, M. \& D. QUANDT. 2014. 20,000 species and five key markers: the status of molecular bryophyte phylogenetics. Phytotaxa 9: 196-228. https://doi.org/10.11646/phytotaxa.9.1.11.

SUÁREZ, G. M. \& M. M. SCHIAVONE. 2009. El género Pilopogon (Bryophyta, Dicranaceae) en la Argentina. Darwiniana 309-314.

SUÁreZ, G. M., M. T. COLOTTI \& M. M. SCHIAVONE. 2005. Dolotortula mniifolia (Sull.) Zand. (Pottiaceae, Musci) en Argentina. Lilloa 42: 81-84.

SUÁREZ, G. M., M. M. SCHIAVONE \& M. T. COLOTTI. 2014. El género Holomitrium (Dicranaceae, Bryophyta), nuevo registro en Argentina y Uruguay. Bol. Soc. Argent. Bot. 49: 457-461. https://doi.org/10.31055/1851.2372.v49.n4.9823

SUÁREZ, G. M \& J. LARRAÍN. 2019. The genus Aongstroemia in Latin America (Dicranaceae, Bryophyta) with special reference to Aongstroemia gayana. Phytotaxa 405: 290-296. https://doi.org/10.11646/phytotaxa.405.6.2

VANDERPOORTEN, A., \& L. HEDENÄS. 2009. New combinations in the Amblystegiaceae. J. Bryol. 31: 129-132. https://doi.org/10.1179/174328209X421845 